\title{
Study on a Single-Axis Fabry-Perot Fiber-Optic Accelerometer and its Signal Demodulation Method
}

\author{
Yun-Feng Liu'Bin Li \\ Institute of Optics and Electronics, \\ No.209 Institute of North Industries Corporation, \\ Chengdu, China \\ E-mail: footballliu@163.com
}

\author{
Shu-Xin Liu \\ No.209 Institute of North Industries Corporation, \\ Chengdu, China \\ E-mail: shuxin0377@163.com
}

\begin{abstract}
A Fabry-Perot fiber-optic accelerometer based on the principle that the acceleration can be sensed through changing the length of the Fabry-Perot cavity by an inertial mass is designed and the Fizeau interferometer based demodulation principle and system for the designed Fabry-Perot fiber-optic accelerometer are proposed. Aiming at the Fizeau interferometer based demodulation principle and system for the designed Fabry-Perot fiber-optic accelerometer, the relative light intensity output from the Fizeau interferometer as a function of the length of the Fabry-Perot cavity and the wedge thickness of the Fizeau interferometer is established. In addition, the theoretical acceleration demodulation model for the Fabry-Perot fiber-optic accelerometer based on a Fizeau interferometer is also established. On these bases, the principle that the acceleration from the Fabry-Perot fiber-optic accelerometer can be demodulated based on the Fizeau interferometer is numerically simulated. The research results indicates the consistency of the simulated results with the theoretical analysis and the feasibility of the acceleration demodulation of the Fabry-Perot fiber-optic accelerometer based on the Fizeau interferometer.
\end{abstract}

Keywords-component; Fabry-Perot fiber-optic sensor, fiberoptic accelerometer, Fizeau interferometer, demodulation

\section{INTRODUCTION}

Fiber-optic accelerometers have been widely used in vibration test, seismic monitoring, aerospace, and other fields $[1,2]$. Comparison with other accelerometers, fiber-optic accelerometers possess the following advantages, including the small size, high immunity to electromagnetic interference, good ability to operate in a wide range of environments, high sensitivity, and good potential for multiplexing [3]. According to operation principles, fiber-optic accelerometers can be categorized as the intensity modulation fiber-optic accelerometers and phase modulation fiber-optic accelerometers. Phase modulation fiber-optic accelerometers can be further classified into Fabry-Perot fiber-optic accelerometers [4], Michelson fiber-optic accelerometers [5], and Mach-Zehnder fiber-optic accelerometers [6]. Because of the small size, large dynamic range, high precision, and long-term stability, Fabry-Perot fiber-optic accelerometers especially fit long-term monitoring occasions. Gerges et al [4] first proposed and studied the principle of a Fabry-Perot fiber-optic accelerometer based on that the acceleration can be sensed through changing the length of the Fabry-Perot cavity by an inertial mass. Preliminary research results indicated that the Fabry-Perot fiber-optic accelerometer can achieve a large dynamic range with high resolution. However, only the preliminary analysis on the sensing phenomenon and performance of the Fabry-Perot fiber-optic accelerometer were presented and no demodulated acceleration time histories of the Fabry-Perot fiber-optic accelerometer were provided. Up to now, no mature signal demodulation schemes for Fabry-Perot fiber-optic accelerometers can be provided.

The commonly used demodulation methods for FabryPerot fiber-optic sensors include the intensity demodulation method and phase demodulation method [7]. Although the intensity demodulation method can be easily realized with low cost, the intensity is susceptible to light intensity fluctuations in the light source, which leads to its low accuracy. Phase demodulation method includes the fringecounting [8], Fourier transforms [9], discrete cavity length transform [10], and Fizeau interferometer based demodulation [11, 12]. The fringe-counting, Fourier transform, and discrete cavity length transform demodulation methods need expensive light spectrum equipments [8-10], which will result in low demodulation speed, so that these schemes cannot be used in high-frequency conditions. Because the Fizeau interferometer based demodulation method does not require expensive broadband light sources and expensive light spectrum equipments, it can be realized with low cost. In addition, the Fizeau interferometer based demodulation method can be realized without moving elements, the Fizeau interferometer based demodulation systems can achieve long-term stability with high accuracy [11]. The above-mentioned demodulation methods have been widely used to demodulate Fabry-Perot fiber-optic sensors for strain and temperature measurement with low-frequency. However, the Fizeau interferometer based demodulation method and system for Fabry-Perot fiber-optic accelerometers with high-frequency have not been reported.

In this paper, a Fabry-Perot fiber-optic accelerometer based on the principle that the acceleration can be sensed through changing the length of the Fabry-Perot cavity by an inertial mass is designed and the Fizeau interferometer based demodulation principle and system for the designed FabryPerot fiber-optic accelerometer are proposed. Aiming at the Fizeau interferometer based demodulation principle and system for the designed Fabry-Perot fiber-optic accelerometer, the relative light intensity output from the Fizeau interferometer as a function of the length of the 
Fabry-Perot cavity and the wedge thickness of the Fizeau interferometer is established. In addition, the theoretical acceleration demodulation model for the Fabry-Perot fiberoptic accelerometer based on a Fizeau interferometer is also established. On these bases, the principle that the acceleration from the Fabry-Perot fiber-optic accelerometer can be demodulated based on a Fizeau interferometer is numerically simulated.

\section{FIZEAU INTERFEROMETER BASED DEMODULATION PRINCIPLE FOR FABRY-PEROT FIBER-OPTIC ACCELEROMETER}

The operation principle and photograph of the developed Fabry-Perot fiber-optic accelerometer are shown in Figures 1(a) and 1(b), respectively. According to Figure 1(a), the developed Fabry-Perot fiber-optic accelerometer is based on the principle that the acceleration can be sensed through changing the length of the Fabry-Perot cavity by an inertial mass. Observing Figure 1, the Fabry-Perot fiber-optic accelerometer comprises a collimation lens, vibrating diaphragm, plane reflector, and inertial mass attached to the vibrating diaphragm. The inertial mass attached to the vibrating diaphragm acts as the acceleration-sensitive element. When acceleration excitation is applied to the accelerometer, the distance between the collimation lens and plane reflector, which equals the length of the Fabry-Perot cavity, will be changed by the inertial mass. The variation of the length of the Fabry-Perot cavity will result in the phase shift of the interference light backed from the single-mode fiber, which will be used to sense the acceleration. According to Figure 1(a), the dynamic length of the FabryPerot cavity of the Fabry-Perot fiber-optic accelerometer is

$$
L=L_{0}+\Delta L
$$

where $L_{0}$ is the static length of the Fabry-Perot cavity of the Fabry-Perot fiber-optic accelerometer without acceleration and $\Delta L$ is the shift length of Fabry-Perot cavity resulted from the acceleration.

According to [4], the acceleration is proportional to the variation of the length of the Fabry-Perot cavity, so that the relationship between the variation of the length of the FabryPerot cavity and the acceleration can be written as

$$
\Delta L=k a
$$

where $a$ is the axial linear acceleration and $k$ is the sensitivity of the Fabry-Perot fiber-optic accelerometer determined by the structural parameters of the Fabry-Perot fiber-optic accelerometer. For the developed accelerometer shown in Figure 1, $k$ is a function of the structural parameters given by

$$
k=\frac{3 m r^{2}\left(1-v^{2}\right)}{\pi E h^{3}}\left[\frac{\left(\frac{r}{b}\right)^{2}-1}{4\left(\frac{r}{b}\right)^{2}}-\frac{\ln ^{2}\left(\frac{r}{b}\right)}{\left(\frac{r}{b}\right)^{2}-1}\right]
$$

where $m$ is the inertial mass; $b$ is the radius of the circular mass; $r, h, E$, and $v$ are the effective compaction radius, thickness, modulus of elasticity, and Poisson ratio of the diaphragm, respectively. According to Equation (2), the acceleration $(a)$ can be assessed if the variation of the length of the Fabry-Perot cavity ( $\Delta L$ ) can be obtained.

According to the operation principle and structure of the developed Fabry-Perot fiber-optic accelerometer as shown in Figure 1, the Fizeau interferometer based demodulation principle and system for the designed Fabry-Perot fiber-optic accelerometer are proposed as shown in Figure 2. According to Figure 2, the light from a broadband light source with a single-mode fiber pigtail is coupled to a four-port coupler through an optical isolator and the transmitted light in one port of the coupler is incident upon the Fabry-Perot fiberoptic accelerometer. The reflected light from the Fabry-Perot fiber-optic accelerometer is incident upon the collimation lens and the Fizeau interferometer through the four-port coupler. The transmitted light from the Fizeau interferometer is monitored with a linear array CCD and the acceleration can be obtained by the signal processing circuit. In fact, the Fizeau interferometer based demodulation is the cavity length matched demodulation and the crucial device is an optical wedge as shown in Figure 3. According to the correlative principle, the transmitted light intensity from the Fizeau interferometer will appear the maximum value when the wedge thickness of the Fizeau interferometer equals the length of the Fabry-Perot cavity, so that the cavity length ( $L$ ) of the Fabry-Perot fiber-optic accelerometer can be assessed according to the position of the maximum value of the light intensity. According to Equations (2) and (3), the acceleration $(a)$ can be demodulated.

Consider the reflectivity of the two surfaces of the Fizeau interferometer, as shown in Figure 3, are equal. When the light with wavelength of $\lambda$ is incident upon the Fizeau interferometer, the intensity of the transmitted light from the wedge at thickness $I(x)$ can be described as ${ }^{[13]}$

$$
I_{\mathrm{t}}(l, \lambda)=\frac{\left(1-R_{\mathrm{F}}\right)^{2}}{1+R_{\mathrm{F}}{ }^{2}-2 R_{\mathrm{F}} \cos \frac{4 \pi l(x)}{\lambda}} I_{\mathrm{r}}(L, \lambda)
$$

where the subscript “ $t$ ” represents transmitted light from the Fizeau interferometer; $R_{\mathrm{F}}$ is the reflectivity of the two surfaces of the Fizeau interferometer; $I_{\mathrm{r}}(L, \lambda)$ is the intensity of the reflected light from the Fabry-Perot fiberoptic accelerometer; $l(x)$ is the wedge thickness at $X$, where the coordinate origin is located at the vertex of the Fizeau interferometer, so that

$$
l(x)=x \sin \theta
$$

where $\theta$ is the angle of the Fizeau interferometer. 


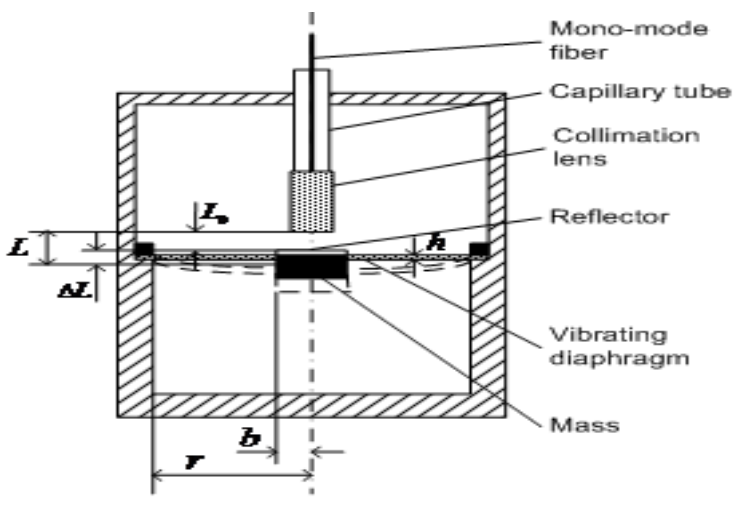

(a)

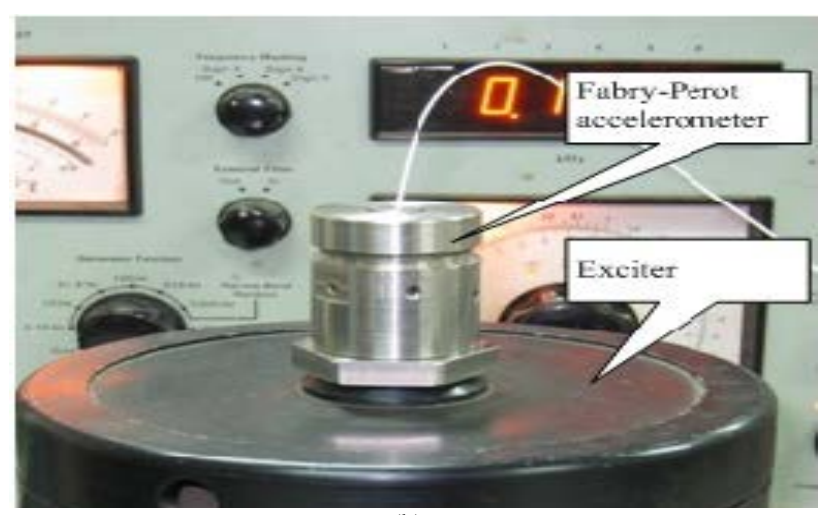

(b)

Figure 1. Developed Fabry-Perot fiber-optic accelerometer: (a) the schematic and (b) the photograph.

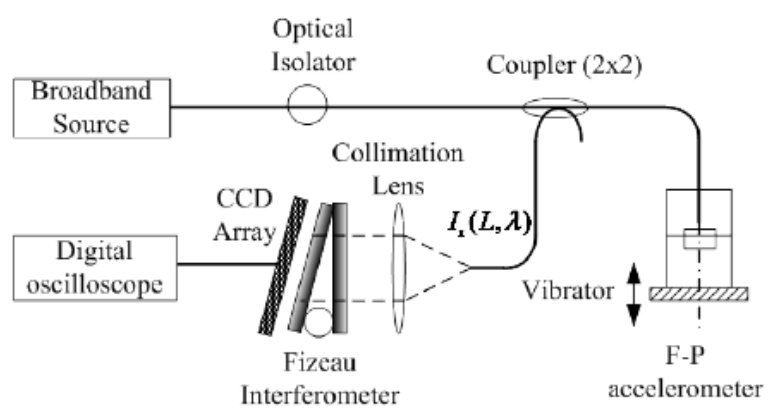

Figure 2. Schematic of the demodulation system for the Fabry-Perot fiberoptic accelerometer based on a Fizeau interferometer.

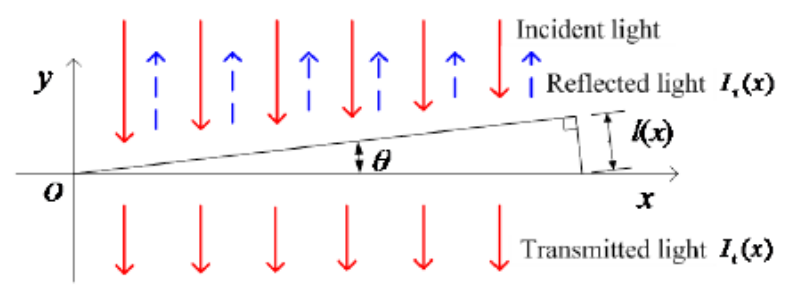

Figure 3. Principle of the Fizeau interferometer.

Consider the reflectivity of the two surfaces of the FabryPerot cavity is $R_{\mathrm{FP}}$. The intensity of the reflected light from the Fabry-Perot fiber-optic accelerometer is given by [11]

$$
I_{\mathrm{r}}(L, \lambda)=\frac{2 R_{\mathrm{FP}}\left[1-\cos \left(\frac{4 \pi}{\lambda} L\right)\right]}{1+R_{\mathrm{FP}}^{2}-2 R_{\mathrm{FP}} \cos \left(\frac{4 \pi}{\lambda} L\right)} I_{\mathrm{s}}(\lambda)
$$

where $L$ is the dynamic length of the Fabry-Perot cavity, which is given by Equation (1), and $I_{s}(\lambda)$ is the intensity of the incident light of the Fabry-Perot fiber-optic accelerometer.

According to [11], the transmitted light intensity from the Fizeau interferometer can be written as

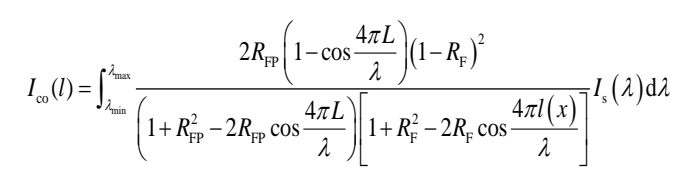

where the subscript " $\mathrm{CO}$ ” represents the coherent light; $\lambda_{\min }$ and $\lambda_{\max }$ represent the minimum and maximum wavelength of the broadband light source, respectively.

In order to facilitate the discussion, the relative light intensity is defined as

$$
I_{0}(l)=\frac{I_{\mathrm{co}}(l)}{I_{\mathrm{s}}(\lambda)}
$$

When $R_{\mathrm{FP}}$ and $R_{\mathrm{F}}$ take a small value $\left(R_{\mathrm{FP}}<<1\right.$ and $R_{\mathrm{F}}<<1$ ), the multi-beam interference can be considered as the two-beam interference. The relative light intensity can be rewritten as

$$
I_{\mathrm{o}}(l)=\int_{\lambda_{\min }}^{\lambda_{\max }}\left(2 R_{\mathrm{FP}}-2 R_{\mathrm{FP}} \cos \frac{4 \pi L}{\lambda}\right)\left[\left(1-2 R_{\mathrm{F}}\right)-2 R_{\mathrm{F}} \cos \frac{4 \pi l}{\lambda}\right] \mathrm{d} \lambda
$$

If we let $R_{\mathrm{FP}}=R_{\mathrm{F}}, A=2 R_{\mathrm{F}}, B=-2 R_{\mathrm{F}}, C=1-2 R_{\mathrm{F}}$, $I_{1}=\int_{\lambda_{\min }}^{\lambda_{\max }} A C \mathrm{~d} \lambda \quad, \quad I_{2}(L)=\int_{\lambda_{\min }}^{\lambda_{\max }} B C \cos \frac{4 \pi L}{\lambda} \mathrm{d} \lambda \quad$, $I_{3}(l)=\int_{\lambda_{\min }}^{\lambda_{\max }} A B \cos \frac{4 \pi l}{\lambda} \mathrm{d} \lambda, \quad I_{4}(L, l)=\frac{B^{2}}{2} \int_{\lambda_{\min }}^{\lambda_{\max }} \cos \frac{4 \pi(L+l)}{\lambda} \mathrm{d} \lambda$, and $I_{5}(L, I)=\frac{B^{2}}{2} \int_{\lambda_{\min }}^{\lambda_{\max }} \cos \frac{4 \pi(L-l)}{\lambda} \mathrm{d} \lambda$, Equation (9) can be rewritten as

$$
I_{0}(l)=I_{1}+I_{2}(L)+I_{3}(l)+I_{4}(L, l)+I_{5}(L, l)
$$

Equation (10) defines the relative light intensity output from the Fizeau interferometer as a function of the length of the Fabry-Perot cavity and the wedge thickness of the Fizeau interferometer. The relationships between the relative light 
intensity given by individual terms of the right-hand side of Equation (10) and the wedge thickness are shown in Figures 4(a) to 4(e), respectively. In Figure 4(f), the relationship between the total relative light intensity and the wedge thickness is shown. According to Figures 4(a) and 4(b), the relative light intensity given by the first two terms $\left(I_{1}\right.$ and $\left.I_{2}\right)$ don't vary with the increase of the wedge thickness $(l)$, that is to say, the first two terms in the right-hand side of Equation (10) are independent of the wedge thickness $(l)$. According to Figure 4(c), the third term $\left(I_{3}\right)$ not only gets the maximum value when $l=0$, but also decreases with the increase of $l$. The equivalent cavity length of the fourth term $\left(I_{4}\right)$ is summation of the Fabry-Perot cavity length $(L)$ and the wedge thickness $(l)$ of the Fizeau interferometer and the equivalent cavity length is larger than either of $L$ and $l$, which determines that the equivalent cavity length is larger than the coherence length of the light source, so that the interference fringes of the fourth term $\left(I_{4}\right)$ are much less than the interference fringes of the third term in the righthand side of Equation (10) and the fourth term $\left(I_{4}\right)$ easily enters the white state of non-interference with no apparent interference fringes as shown in Figure 4(d). Figure 4(e) shows the relationship between the fifth term $\left(I_{5}\right)$ and $l$. The equivalent cavity length of the fifth term $\left(I_{5}\right)$ is the difference between the length of the Fabry-Perot cavity $(L)$ and the wedge thickness $(l)$ of the Fizeau interferometer and $I_{5}$ gets the maximum value when $l=L$. The fifth term is the decisive factor of the demodulation system. Figure 4(f) illustrates the relationship between the total relative light intensity given by Equation (10) and the wedge thickness and " $\mathrm{P}$ " represents the peak value of the relative light intensity output from the Fizeau interferometer. According to figure 4(f), the maximum value of $I_{\mathrm{o}}(l)$ appears at $l=0$ and $l=L$ as shown in Figures 4(c) and 4(e). The maximum value of $I_{0}(l)$ at $l=0$ reflects the unilateral attenuation of the interference fringes from the third term $\left(I_{3}\right)$ and the maximum value at $l=L$ incarnates the symmetric attenuation of interference fringes from the fifth term $\left(I_{5}\right)$. The effective location $\left(\left.x\right|_{\operatorname{MAX}\left[I_{0}(l)\right]}\right)$ of the maximum value of the light intensity appears at $l=L$, where the wedge thickness of the Fizeau interferometer is equal to the length of the FabryPerot cavity and is given by

$$
L=l\left(\left.x\right|_{\operatorname{MAX}\left[I_{0}(l)\right]}\right)
$$

According to Equations (1), (5), and (11), the cavity length shift of the Fabry-Perot fiber-optic accelerometer is given by

$$
\Delta L=\left.x\right|_{\operatorname{MAX}\left[I_{0}(l)\right]} \sin \theta-L_{0}
$$

Equation (12) defines the relationship between the cavity length shift of the Fabry-Perot fiber-optic accelerometer and the position of the maximum value of the light intensity on the Fizeau interferometer. According to Equations (2) and (12), the relationship between the acceleration $a$ and the location $\left.x\right|_{\operatorname{MAX}\left[I_{0}(l)\right]}$ of the maximum value of the light intensity on the Fizeau interferometer is given by

$$
a=\frac{\left.x\right|_{\operatorname{MAX}\left[I_{0}(l)\right]} \sin \theta-L_{0}}{k}
$$

The theoretical acceleration demodulation model for the Fabry-Perot fiber-optic accelerometer based on Fizeau interferometer is given by Equations (3) and (13). According to Equation (13), the acceleration ( $a$ ) can be demodulated when the position $\left(\left.x\right|_{\operatorname{MAx}\left[I_{0}(l)\right]}\right)$ of the maximum value of the light intensity can be obtained. 


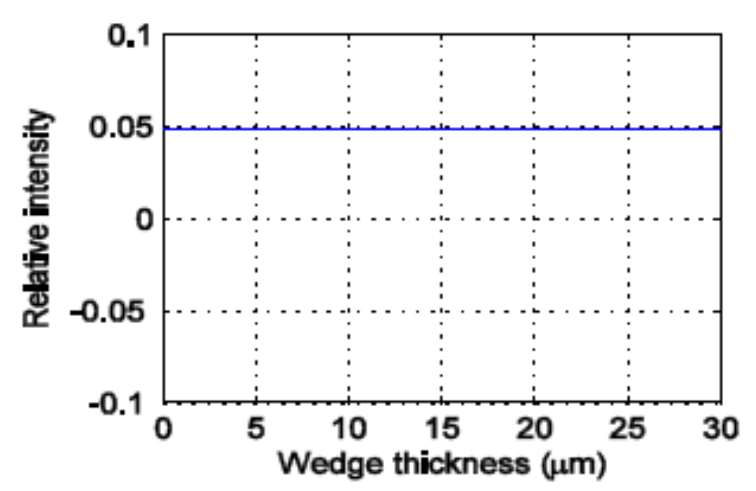

(a)

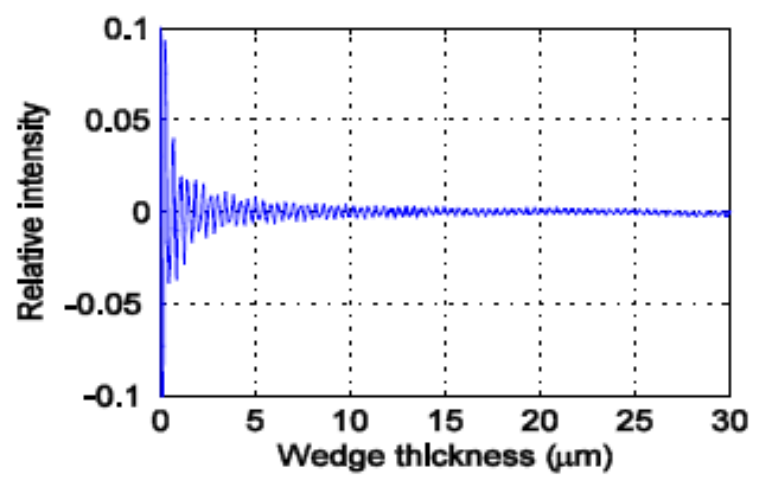

(c)

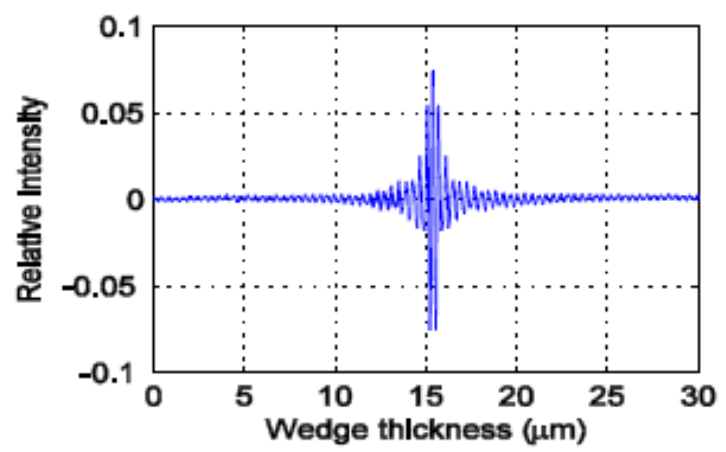

(e)

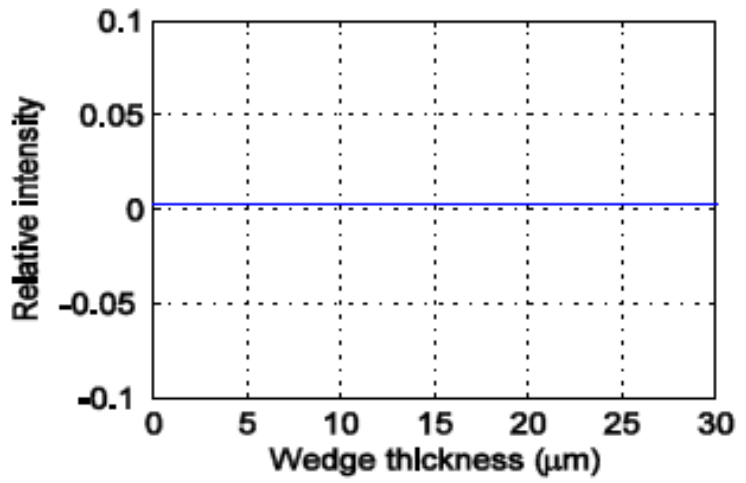

(b)

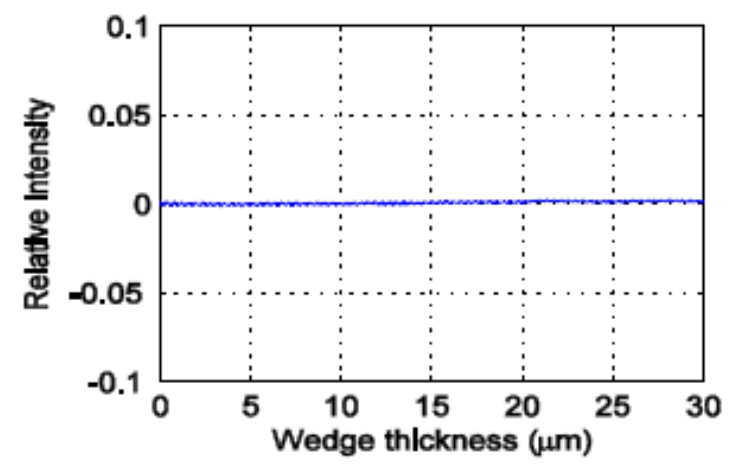

(d)

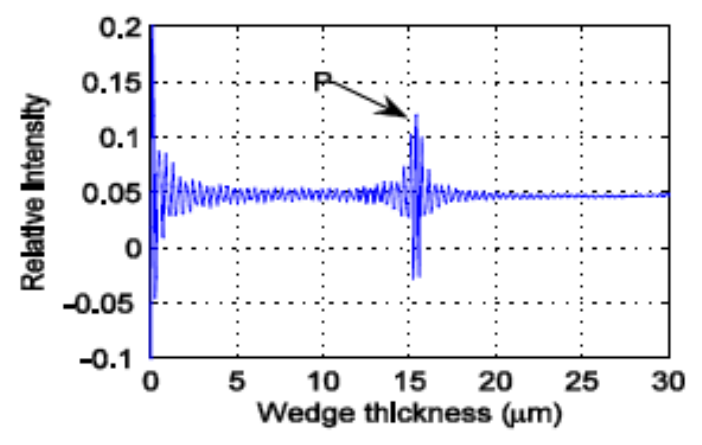

(f)

Figure 4. Relationships between the individual terms in the right-hand side of Equation (10) and the wedge thickness: (a) the relationship between and the wedge thickness, (b) the relationship between and the wedge thickness, (c) the relationship between and the wedge thickness, (d) the relationship between and the wedge thickness, (e) the relationship between and the wedge thickness, and (f) the relationship between and the wedge thickness.

\section{NUMERICAL RESULTS AND DISCUSSION}

Assuming $_{L_{0}}=15 \mu \mathrm{m}, k=0.5 \mu \mathrm{m} / \mathrm{g}$, and $\theta=0.05^{\circ}$ of the designed Fabry-Perot fiber-optic accelerometer, the principle that the acceleration sensed by the Fabry-Perot fiber-optic accelerometer can be demodulated based on the Fizeau interferometer is numerically simulated used matlab when an impulse excitation and a $100 \mathrm{~Hz}$ sinusoidal excitation with amplitude of $2 \mu \mathrm{m}$ and phase of $\pi / 4$ are applied to the designed Fabry-Perot fiber-optic accelerometer, respectively.
The numerical results of the emergent light demodulated based on the Fizeau interferometer are shown in Figures 5 and 6 , respectively.

The relationships between the demodulated relative light intensity from the Fabry-Perot fiber-optic accelerometer and the wedge thickness are shown in Figure 5 when the FabryPerot fiber-optic accelerometer is applied an impulse acceleration excitation at $0.01 \mathrm{~s}$. Figure 5(a) shows the relative light intensity from the Fizeau interferometer as a function of the time and the wedge thickness of the Fizeau 
interferometer. The position time history of the maximum value of the light intensity from the Fizeau interferometer is shown in Figure 5(b). Figures 5(c), 5(d), and 5(e) show the relationships between the relative light intensity demodulated by the Fizeau interferometer and the wedge thickness at $0.0095 \mathrm{~s}, 0.01 \mathrm{~s}$, and $0.0105 \mathrm{~s}$, respectively. According to Figures 5(c), 5(d), and 5(e), the maximum value of the light intensity appears when $l=15 \mu \mathrm{m}$ at $0.0095 \mathrm{~s}$, the position of the maximum value of the light intensity appears when $l=17$ $\mu \mathrm{m}$ at $0.01 \mathrm{~s}$, and the position of the maximum value of the light intensity appears when $l=15 \mu \mathrm{m}$ at $0.0105 \mathrm{~s}$. It is clear that the acceleration sensed by the Fabry-Perot fiber-optic accelerometer excited by an impulse excitation at $0.01 \mathrm{~s}$ can be demodulated based on the Fizeau interferometer. Figure 5(f) shows the demodulated acceleration time history of the Fabry-Perot fiber-optic accelerometer. According to Figure 5(f), the acceleration sensed by the Fabry-Perot fiber-optic accelerometer is $4 \mathrm{~g}$ at $0.01 \mathrm{~s}$, which equals the acceleration applied to the Fabry-Perot fiber-optic accelerometer.

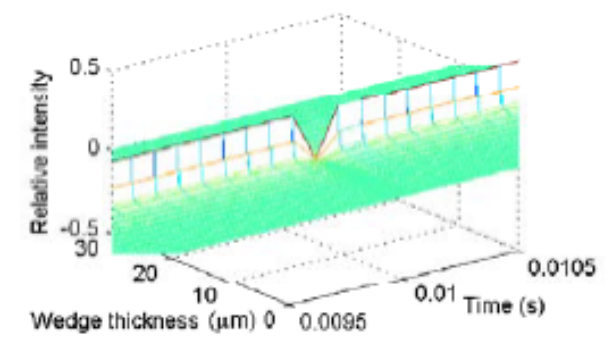

(a)

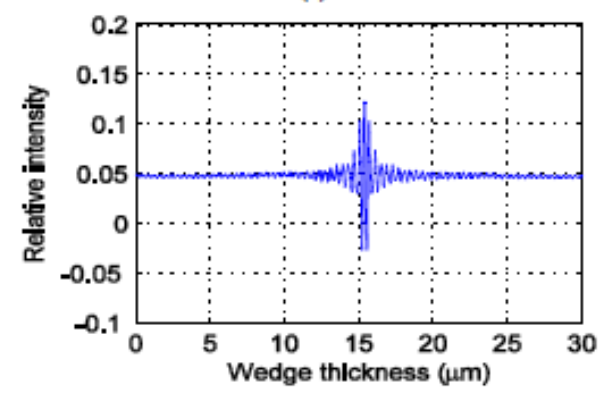

(c)

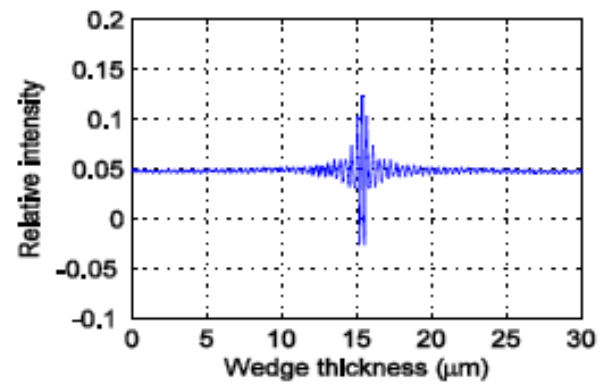

(e)
Figure 6 illustrates the demodulated relative light intensity from the Fizeau interferometer when the FabryPerot fiber-optic accelerometer is excited by a $100 \mathrm{~Hz}$ sinusoidal excitation with amplitude of $2 \mu \mathrm{m}$ and initial phase of $\pi / 4$. The relative light intensity from the Fizeau interferometer as a function of the time and the wedge thickness is shown in Figure 6(a). According to Figure 6(a), the maximum value of the relative light intensity demodulated by the Fizeau interferometer matchs well the length of the Fabry-Perot cavity with the sinusoidal excitation. Figure 6(b) shows the demodulated acceleration time history of the Fabry-Perot fiber-optic accelerometer. According to Figure 6(b), the demodulated acceleration time history by the Fizeau interferometer is a $100 \mathrm{~Hz}$ sinusoidal acceleration with amplitude of $0.0806 \mathrm{~g}$ and initial phase of $\pi / 4$, which equals the applied acceleration excitation.

According to Figures 5 and 6, the Fizeau interferometer can be used to accurately demodulate the acceleration for the designed Fabry-Perot fiber-optic accelerometer.

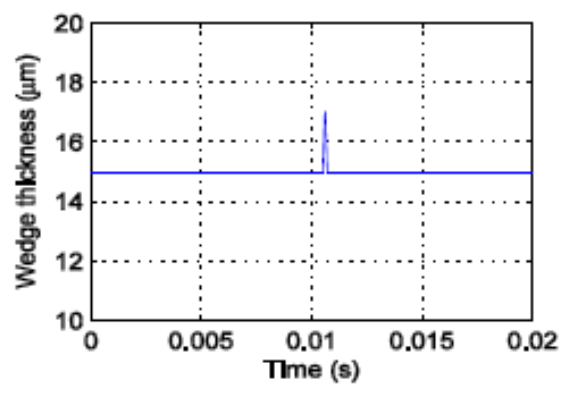

(b)

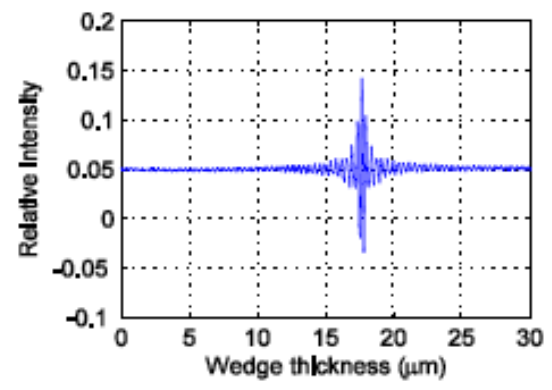

(d)

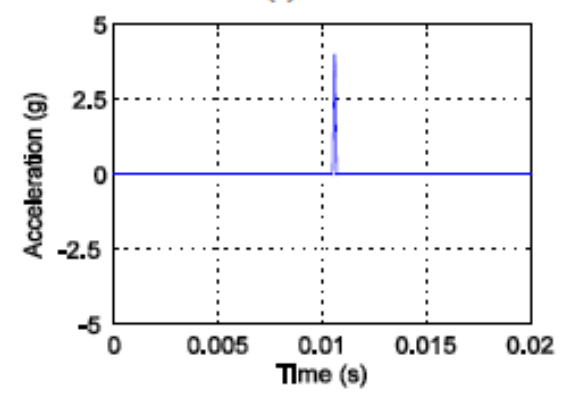

(f)

Figure 5. The output from the Fizeau interferometer when the Fabry-Perot fiber-optic accelerometer is excited by an impulse excitation: (a) the relative light intensity from the Fizeau interferometer as a function of the time and the wedge thickness, (b) the position time history of the maximum value of the light intensity from the Fizeau interferometer, (c) the relationship between the relative light intensity at $0.0095 \mathrm{~s}$ and the wedge thickness, (d) the relationship between the relative light intensity at $0.01 \mathrm{~s}$ and the wedge thickness, (e) the relationship between the relative light intensity at $0.0105 \mathrm{~s}$ and the wedge thickness, and (f) the demodulated acceleration time history of the Fabry-Perot fiber-optic accelerometer. 


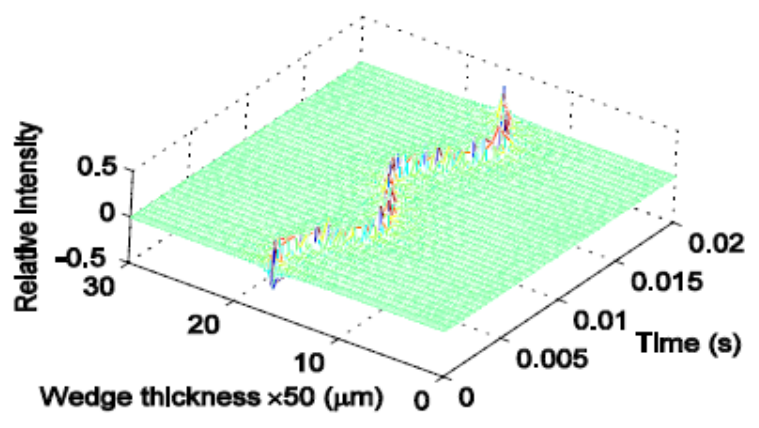

(a)

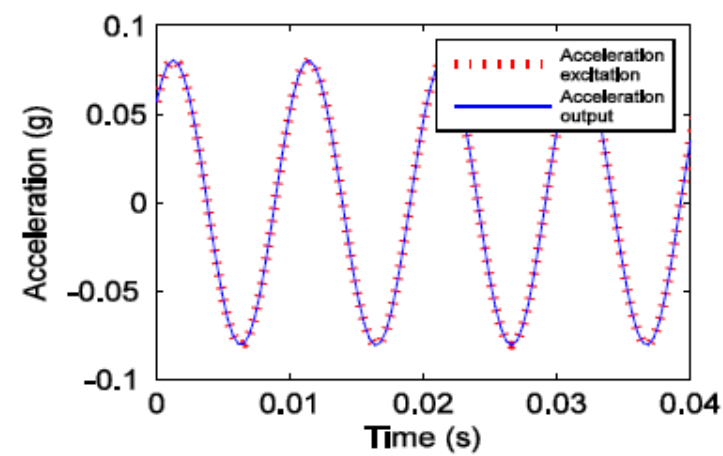

(b)

Figure 6. The output from the Fizeau interferometer when the Fabry-Perot fiber-optic accelerometer is excited by a $100 \mathrm{~Hz}$ sinusoidal excitation with amplitude of $2 \mu \mathrm{m}$ and initial phase of $\pi / 4$ : (a) the relative light intensity of the Fizeau interferometer as a function of the time and the wedge thickness and (b) the demodulated acceleration time histories of the Fabry-Perot fiber-optic accelerometer.

\section{CONCLUSIONS}

In this paper, a Fabry-Perot fiber-optic accelerometer based on the principle that the acceleration can be sensed through changing the length of the Fabry-Perot cavity by an inertial mass was designed and developed and the demodulation principle and system based on a Fizeau interferometer for the designed and developed Fabry-Perot fiber-optic accelerometer were proposed. Aiming at the demodulation principle and system based on a Fizeau interferometer for the designed Fabry-Perot fiber-optic accelerometer, the relative light intensity output from the Fizeau interferometer as a function of the length of FabryPerot cavity and the wedge thickness of the Fizeau interferometer was established. In addition, the theoretical acceleration demodulation model for the Fabry-Perot fiberoptic accelerometer based on a Fizeau interferometer was also established. On these bases, the principle that the acceleration from the Fabry-Perot fiber-optic accelerometer can be demodulated based on the Fizeau interferometer was numerically simulated. The research results indicated the consistency of the simulated results with the theoretical analysis and the feasibility of the acceleration demodulation of the Fabry-Perot fiber-optic accelerometer based on the Fizeau interferometer.

\section{REFERENCES}

[1] Lee B, 2003, Review of the present status of optical fiber sensors, Optical Fiber Technology, Vol. 9, No. 2, 57-79

[2] Ni Xiaohong, Gui Feifei, Wang Yutian, et al. Simultaneous measurement of temperature and strain using fiber grating $[\mathrm{J}]$ (in Chinese). Infrared and Laser Engineering, 2011, 40(7): 724-728..
[3] Zeng N, Shi C Z, Zhang M, Wang L W, Liao Y B, and Lai S R, 2004, A 3-component fiber-optic accelerometer for well logging, Optics Communication, Vol. 234, No. 1-6, 153-162

[4] Gerges A S, Newson T P, Jones J D C, and Jackson D A, 1989, Highsensitivity fiber-optic accelerometer, Optics Letters, Vol. 14, No. 4, 251-253

[5] Chen C H, Ding G L, Zhang D L, and Cui Y M, 1998, Michelson fiber optic accelerometer, Review of Scientific Instruments, Vol. 69, No. 9, 3123-3126

[6] Pannell C N, Jones J D C, and Jackson D A, 1994, The effect of environmental acoustic nosie on optical fiber based velocity and vibration sensor systems, Measurement Science and Technology, Vol. 5, No. 4, 412-417

[7] Sun J Y, Chen W M, Zhu Y, et al. 2002, An optic fiber Fabry Perot strain sensor system based on tunable Fabry Perot (in Chinese), Laser Journal, Vol. 23, No. 4, 49-50.

[8] Chang C C and Sirkis J, 1996, Absolute phase measurement with extrinsic Fabry-Perot optical fiber sensors, Proceedings of the SPIE conference on Fiber Optic and Laser Sensors, Vol. 2839, pp. 111-121

[9] Liu T and Frenando G F, 2000, A frequency division multiplexed low-finesse fiber optic Fabry-Perot sensor system for strain and displacement measurements, Review of Scientific Instruments, Vol. 71, No. 3, 1275-1287

[10] Chen Y S, 2003, Algorithms and multiplexing of fiber Fabry-Perot strain sensor (in Chinese), Master thesis, Chongqing University, Chongqing, China, pp. 16-20.

[11] Zhang P, Zhu Y, Lei X H, and Chen W M, 2004, Cross-correlation demodulation of optic fiber Fabry-Perot sensor multiplexed in parallel (in Chinese), Opto-electronic Engineering, Vol. 31, No. 11, 70-72.

[12] Fiso Technologies Inc. 1997, Fiber Optic Strain Gauge Installation Guide

[13] Belleville C and Duplain G, 1993, White-light interferometric multimode fiber-optic strain sensor, Optics Letters, Vol. 18, No. 1, 78-80 